\title{
Different Periods of Periconceptional Undernutrition Have Different Effects on Growth, Metabolic and Endocrine Status in Fetal Sheep
}

\author{
CHRISTOPHER W. H. RUMBALL, FRANK H. BLOOMFIELD, MARK H. OLIVER, AND JANE E. HARDING \\ Liggins Institute, University of Auckland, Private Bag 92019, Auckland, New Zealand
}

\begin{abstract}
Periconceptional undernutrition alters fetal growth and development. However, there are no data on separate effects of undernutrition before and after conception and few on underlying mechanisms. We determined the effects of mild periconceptional undernutrition on late gestation fetal growth, glucose-insulin axis, and maternal and fetal hypothalamic-pituitary-adrenal axes. Ewes were undernourished for $60 \mathrm{~d}$ before conception, $30 \mathrm{~d}$ after, or both, compared with well-nourished controls. Undernutrition before conception resulted in smaller, slower-growing fetuses with relatively larger placentae. Ewes that gained weight before, but lost weight after mating, or vice versa, had the smallest fetuses. Fetuses of ewes undernourished only before conception grew more slowly following instrumentation, and fetuses in both preconception undernutrition groups slowed their growth with a maternal fast. The fetal glucoseinsulin axes and maternal and fetal hypothalamic-pituitary-adrenal axis were not different among groups. Maternal undernutrition at different periods around conception has different effects on fetal growth trajectory that are not reflected in size in late gestation. Preconceptional undernutrition alone alters fetal growth responses to late gestation stressors, suggesting that maternal nutrition is important at both times, and that fetal effects are neither due solely to substrate limitation, nor to excess fetal glucocorticoid exposure at the time of undernutrition. (Pediatr Res 66: 605-613, 2009)
\end{abstract}

$\mathrm{T}$ The link between altered fetal development and the risk of adult diseases is partly mediated by permanent changes in the glucose-insulin axis and hypothalamic-pituitary-adrenal axis (HPAA) and may be accompanied by changes in fetal growth. Maternal nutrition is one mechanism by which prenatal events can influence postnatal disease risk, including macronutrient balance (1), micronutrient (2), and overall caloric (3) intakes. Different timings of undernutrition during pregnancy have different effects $(3,4)$. In particular, undernutrition in early gestation is associated with impaired insulin secretion, obesity, and coronary heart disease (3). Offspring of rats fed a low protein diet in the preimplantation period have altered blood pressure, birth weight, and postnatal growth (5).

In sheep, undernutrition from $60 \mathrm{~d}$ before to $30 \mathrm{~d}$ after mating alters fetal growth trajectory (6) response to maternal undernutrition (7), insulin response to a glucose bolus (8), and

Received March 20, 2009; accepted August 6, 2009.

Correspondence: Jane E. Harding, F.R.A.C.P., D.Phil., Liggins Institute, University of Auckland, Private Bag 92019, Auckland, New Zealand; e-mail: j.harding@ auckland.ac.nz

Supported by grants from the Health Research Council of New Zealand, the National Research Centre for Growth and Development, the Auckland Medical Research Foundation, and the New Zealand Lottery Board. pituitary ACTH response to decreased negative feedback (9). At least some of these endocrine effects persist postnatally (10). Undernutrition from $45 \mathrm{~d}$ before to $7 \mathrm{~d}$ after mating also alters relationships between maternal weight change and uteroplacental weight (11), in addition to fetal adrenal weight, IGF-2, and $\mathrm{P} 40_{\mathrm{c} 17}$ expression (12). Undernutrition from mating to $30 \mathrm{~d}$ alters pituitary, adrenal (13), and cardiovascular function (14) of offspring.

However, none of these studies examined the separate effects of undernutrition in the preconception and postconception periods using the same undernutrition protocol. There are also few data regarding maternal metabolic and HPAA function following periconceptional undernutrition.

The aims of this study were to determine the effects of mild undernutrition of ewes before conception during oocyte maturation, after conception during attachment and placentation, or both, on late gestation fetal growth, glucose-insulin and HPAA, and on the maternal HPAA.

\section{METHODS}

\begin{abstract}
Animals. Ethical approval was obtained from the University of Auckland Animal Ethics Committee. Multiparous 4- to 5-y-old Romney ewes were acclimatized to a concentrate feed (CamTech, Cambridge, NZ), then randomly divided into four groups: controls (C; ad libitum feeds at 3-4\% of body weight per day); undernutrition from $60 \mathrm{~d}$ before to mating [preconception (pre-C)]; undernutrition from $2 \mathrm{~d}$ before mating to $30 \mathrm{~d}$ after [postconception (post-C)]; or undernutrition from $60 \mathrm{~d}$ before to $30 \mathrm{~d}$ after mating (pre+ post-C). Undernutrition comprised a 2-d fast then concentrate feeds individually adjusted to achieve and maintain 10 to $15 \%$ body weight reduction. Feed intake was initially 1 to $2 \%$ of body weight per day, increasing to approximately $80 \%$ of controls. Ewes were fed ad libitum when not undernourished. After ultrasound scanning at $55 \mathrm{~d}$, only singleton-bearing ewes were retained. Blood samples were taken by jugular puncture at regular intervals until transport to the laboratory at $104 \mathrm{~d}$, and ewes were weighed at least twice weekly.

After acclimatization to the laboratory for $5 \mathrm{~d}$, ewes underwent surgery under general anesthesia at $110 \mathrm{~d}$ for placement of maternal and fetal arterial, venous, and amniotic catheters. Growth catheters were fitted around the fetal chest (15). These were measured twice daily, and mean values were used in the analyses.

Baseline maternal and fetal blood samples were taken in the morning before feeding at 114, 117, 121, 127, and $131 \mathrm{~d}$ (Fig. 1). At $118 \mathrm{~d}$, after an overnight fast, a glucose challenge was performed on the fetuses. Glucose $1.5 \mathrm{~g}$ was injected i.v., and arterial blood samples were taken at $0,2,5,10,15,30,45$, and $60 \mathrm{~min}$. Four hours later an arginine challenge was performed. Arginine 300 mg (Sigma Chemical Co. Chemical, St. Louis) was injected i.v. and blood samples were taken at the same intervals.
\end{abstract}

\footnotetext{
Abbreviations: AUC, area under the curve; AVP, arginine vasopressin; $\boldsymbol{\beta H B A}, \beta$-hydroxybutyrate; CRH, corticotropin-releasing hormone; $\mathbf{C V}$, coefficient of variation; HPAA, hypothalamic-pituitary-adrenal axis
} 


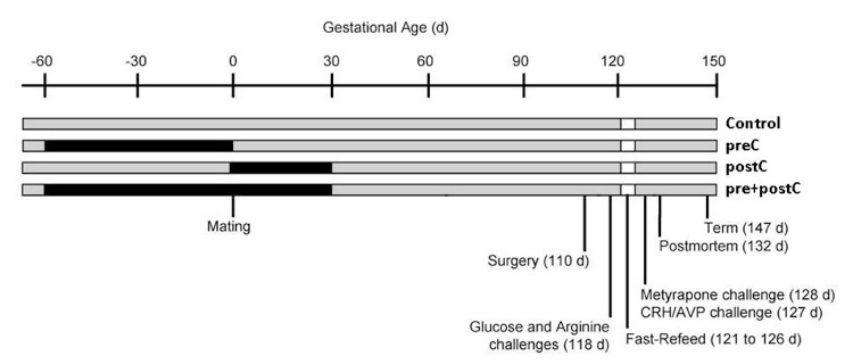

Figure 1. Timeline of experimental protocol. Black bar indicates period of maternal undernutrition and white bar maternal fast.

Ewes were fasted for 3 days (121-124 d) and refed on day 124, while simultaneously receiving i.v. glucose $25 \mathrm{~g}$ over $8 \mathrm{~h}$ to restore blood glucose concentrations in a 'square wave' pattern. Maternal and fetal blood samples were taken daily during fasting and 8,24 , and $48 \mathrm{~h}$ after refeeding.

On day 127, $2.0 \mu \mathrm{g}$ of bovine corticotropin-releasing hormone (CRH) and 0.4 $\mu \mathrm{g}$ arginine vasopressin (AVP) (Sigma Chemical Co.) were injected i.v. to the fetus, and blood samples were taken at $-15,0,15,30,45,60,120$, and $240 \mathrm{~min}$. On day 128, $60 \mathrm{mg}$ metyrapone (Novartis Pharma, Basel, Switzerland) was injected i.v., and blood samples were taken at $-30,0,30,60$, and $120 \mathrm{~min}$. Blood samples were drawn into a tube containing lithium heparin on ice, centrifuged at $4^{\circ} \mathrm{C}$, and the plasma stored at $-80^{\circ} \mathrm{C}$ until assay.

Sheep were killed with an overdose of pentobarbitone at $132 \mathrm{~d}$. The fetus and placenta were dissected, and placentomes were categorized according to morphology (16).

Metabolite and hormone assays. Metabolite concentrations were measured on a Hitachi 902 auto-analyser (Hitachi, North Ryde, Australia): glucose by enzymatic colorimetric assay (Roche, Basel, Switzerland); urea by kinetic UV assay (Roche); lactate and FFA by enzymatic colorimetric assays (Randox Laboratories, Crumlin, UK); and $\beta$-hydroxybutyrate ( $\beta \mathrm{HBA})$ by kinetic UV assay (Randox). Insulin was measured by RIA (17), using ovine insulin as the standard (Sigma Chemical Co., batch no. I9254). IGF-I (18) and ACTH (Diasorin, Stillwater, OK) were measured by RIA, and steroids using mass spectrometry (19).

Data analysis. Metabolite and hormone data were averaged over the preconception period ( -60 to $0 \mathrm{~d}$ ), postconception ( 0 to $30 \mathrm{~d}$ ), midgestation (40 to $97 \mathrm{~d}$ ), late gestation in the laboratory (114 to $121 \mathrm{~d}$ ), and before postmortem (128 to $131 \mathrm{~d}$ ). Growth data were divided into prefasting (114 to $121 \mathrm{~d}$ ), fasting (122 to $124 \mathrm{~d}$ ), and refeeding (125 to $131 \mathrm{~d}$ ). Food intake was compared on days it was unrestricted between 114 and $131 \mathrm{~d}$. Changes in growth rate were assessed using multiple linear regression with a variable delineating prefasting from fasting and group by time interactions as the variables of interest (15).

Interactions between maternal weight and preconception and postconceptional weight changes were explored using multiple linear regression with maternal weight at $-71 \mathrm{~d}$, weight change from -71 to $-2 \mathrm{~d}$, and weight change from 0 to $30 \mathrm{~d}$ as independent variables, including all interactions. For each fetal factor, this model was refined using a forward stepwise regression with a probability to enter of 0.1 . To reduce risk of type I errors, results are only presented where a consistent pattern emerged across related fetal factors.

Data were compared between nutritional groups using ANOVA with fetal sex as a covariate and Tukey-Kramer correction for multiple comparisons. Changes in metabolite and hormone levels between time periods were investigated by matched pairs analysis within groups. For glucose and arginine challenges, area under the curve (AUC) was calculated from baseline levels, and estimated fetal weight (20) was included as a covariate to account for the variable dose relative to fetal weight. For CRH and AVP and metyrapone challenges, AUC was calculated from baseline, taken as the average of the two baseline samples, and the data were log transformed to approximate normality where required. Illustrated AUCs are the least square means, and geometric mean and upper SE if data were log transformed.

Statistical analyses were performed using JMP 5.1 (SAS Institute, Cary, $\mathrm{NC})$. Data are presented as mean \pm SEM.

\section{RESULTS}

Fifty-three singleton-bearing ewes ( 17 control, 12 pre-C, 11 post- $\mathrm{C}$, and 13 pre + post-C) entered the experiment, 41 (10 control, 10 pre-C, 9 post-C, and 12 pre + post-C) completed the fast-refeed protocol, 36 ( 9 control, 8 pre-C, 8 , and 11 pre+ post-C) completed the HPAA challenges, and postmor- tem data were available for 35 ( 9 control, 8 pre-C, 8 post-C, and 10 pre+ post-C).

Maternal weight. Ewe weights were similar among groups at $-71 \mathrm{~d}$ and $110 \mathrm{~d}$ (Table 1, Fig. 2). Control ewes gained weight to be $6 \%$ heavier at $110 \mathrm{~d}$ than at $-71 \mathrm{~d}(p<0.01)$. Pre-C ewes lost $9.0 \%$ of weight during undernutrition, post-C ewes lost $15 \%$, whereas pre + post-C ewes lost $15 \%$ and were still $4 \%$ lighter at $110 \mathrm{~d}$ than at $-71 \mathrm{~d}(p<0.01)$. Food intake in late gestation was higher in these ewes (pre+post-C, $2.23 \pm 0.04 \mathrm{~kg} \cdot \mathrm{d}^{-1} ;$ control, $2.01 \pm 0.05 \mathrm{~kg} \cdot \mathrm{d}^{-1}$; post-C,

Table 1. Maternal weights

\begin{tabular}{lcccl}
\hline & $-71 \mathrm{~d}$ & \multicolumn{1}{c}{$-2 \mathrm{~d}$} & \multicolumn{1}{c}{$30 \mathrm{~d}$} & \multicolumn{1}{c}{$110 \mathrm{~d}$} \\
\hline Control & $64.2 \pm 1.2$ & $65.5 \pm 1.5^{\mathrm{a}}$ & $68.4 \pm 1.5^{\mathrm{a}}$ & $68.0 \pm 1.4^{* *}$ \\
Pre-C & $64.2 \pm 1.3$ & $54.0 \pm 1.2^{\mathrm{b}}$ & $60.6 \pm 1.2^{\mathrm{b}}$ & $65.2 \pm 1.3$ \\
Post-C & $63.7 \pm 1.8$ & $66.8 \pm 2.1^{\mathrm{a}}$ & $56.7 \pm 1.9^{\mathrm{bc}}$ & $63.8 \pm 2.2$ \\
Pre+post-C & $65.0 \pm 1.5$ & $55.2 \pm 1.3^{\mathrm{b}}$ & $54.6 \pm 1.2^{\mathrm{c}}$ & $62.5 \pm 1.6^{* *}$ \\
\hline
\end{tabular}

Data are mean \pm SEM in $\mathrm{kg}$. Different letters within a column indicate significant differences between groups $(p<0.05)$. ** $p<0.01$ for comparison between weights at -71 and $110 \mathrm{~d}$ within the same group.

Pre-C, preconception; Post-C, postconception.

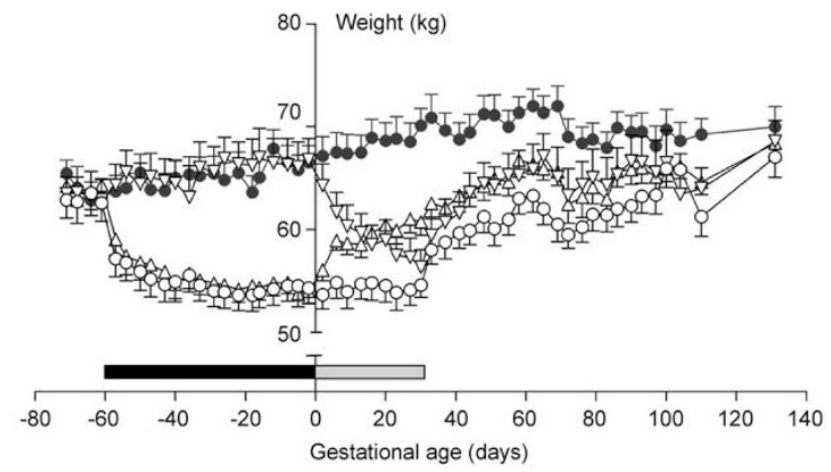

Figure 2. Ewe weights from -71 to $132 \mathrm{~d}$ showing preconception (black bar) and postconception undernutrition (gray bar). Control $\bullet$, pre-C $\triangle$, post-C $\nabla$, and pre+post-C $\bigcirc$. Brief weight loss from $60 \mathrm{~d}$ was because of shearing.

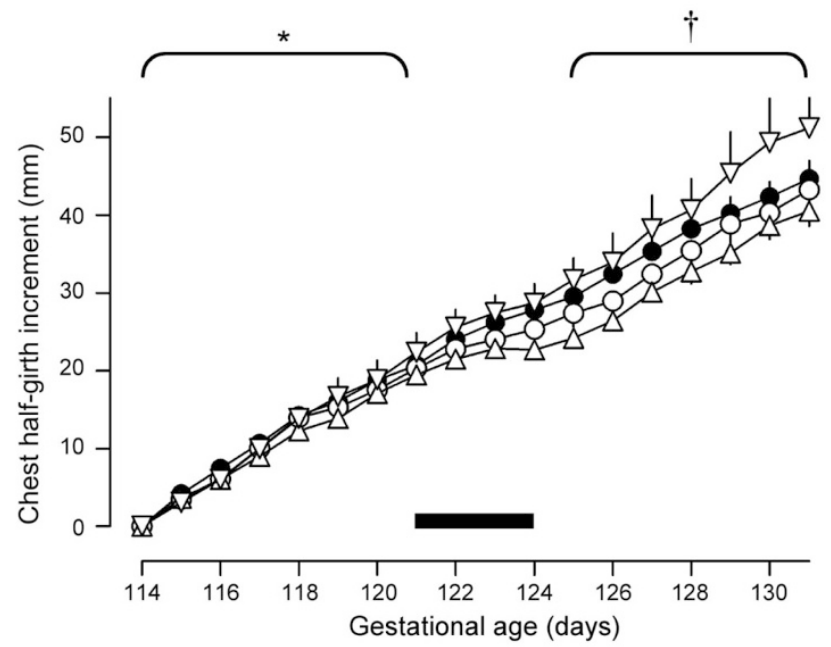

Figure 3. Cumulative fetal chest girth increments. Control $\bullet$, pre-C $\triangle$, post-C $\nabla$, and pre+post-C $\bigcirc$. Ewes fasted from 121 to $124 \mathrm{~d}$ (black bar). $* p<0.05$ for control $v s$ pre-C, $\dagger p<0.05$ for post-C $v s$ all other groups. 
$2.00 \pm 0.05 \mathrm{~kg} \cdot \mathrm{d}^{-1}$, both $p<0.05$; pre-C, $2.18 \pm 0.06$ $\mathrm{kg} \cdot \mathrm{d}^{-1}$, not different).

Fetal growth. Control fetuses tended to slow their growth by $37 \%$ with maternal fasting ( $2.98 \pm 0.16$ to $1.88 \pm 0.47$ $\mathrm{mm} \cdot \mathrm{d}^{-1}, p=0.09$; Fig. 3). Growth rate after refeeding tended to be $17 \%$ slower than before fasting $(2.46 \pm 0.15$ $\left.\mathrm{mm} \cdot \mathrm{d}^{-1}, p=0.08\right)$.

Pre-C fetuses at surgery were similar in size to controls, but they grew more slowly from 114 to $121 \mathrm{~d}(p<0.05)$ and slowed their growth by $78 \%$ with maternal fasting $(2.75 \pm$ 0.10 to $0.60 \pm 0.35 \mathrm{~mm} \cdot \mathrm{d}^{-1}, p<0.01$; Fig. 3). After refeeding, their growth was $6 \%$ slower than before fasting $\left(2.60 \pm 0.18 \mathrm{~mm} \cdot \mathrm{d}^{-1}, p<0.01\right)$ and similar to controls. At postmortem, they tended to have smaller hearts, be lighter than controls, had shorter hind limbs, and smaller fetal-toplacental weight ratio (Table 2).

Post-C fetuses grew at a rate similar to controls but did not significantly slow their growth with maternal fasting (3.23 \pm 0.25 to $1.57 \pm 0.33 \mathrm{~mm} \cdot \mathrm{d}^{-1}, p=0.25$; Fig. 3). After refeeding, their growth was similar to before fasting $\left(3.03 \pm 0.31 \mathrm{~mm} \cdot \mathrm{d}^{-1}\right.$, $p=0.25)$, but greater than controls $(p<0.05)$. They achieved similar size to that of controls at postmortem (Table 2).

Pre + post-C fetuses were similar in size to controls at surgery and grew at a similar rate, but slowed their growth by

Table 2. Fetal morphometric data at surgery and at postmortem

\begin{tabular}{|c|c|c|c|c|}
\hline & Control & Pre-C & Post-C & Pre + post $-\mathrm{C}$ \\
\hline Surgery $111 \pm 1 \mathrm{~d}$ & $(n=18)$ & $(n=12)$ & $(n=11)$ & $(n=14)$ \\
\hline Chest girth $(\mathrm{cm})$ & $27.0 \pm 0.4$ & $26.2 \pm 0.6$ & $25.4 \pm 0.6$ & $26.1 \pm 0.7$ \\
\hline Hindlimb length $(\mathrm{cm})$ & $25.9 \pm 0.4$ & $25.1 \pm 0.3$ & $25.5 \pm 0.5$ & $25.5 \pm 0.3$ \\
\hline Post mortem 132 d & $(n=9)$ & $(n=8)$ & $(n=8)$ & $(n=10)$ \\
\hline Fetal weight $(\mathrm{g})$ & $4391 \pm 147^{\mathrm{A}}$ & $3851 \pm 173^{\mathrm{B}}$ & $4445 \pm 145^{\mathrm{A}}$ & $4332 \pm 141^{\mathrm{AB}}$ \\
\hline Chest girth $(\mathrm{cm})$ & $34.6 \pm 0.6^{\mathrm{ab}}$ & $32.8 \pm 0.5^{\mathrm{b}}$ & $34.8 \pm 0.5^{\mathrm{ab}}$ & $35.5 \pm 0.8^{\mathrm{a}}$ \\
\hline Crown-rump length $(\mathrm{cm})$ & $46.9 \pm 1.0$ & $45.1 \pm 0.7$ & $46.0 \pm 1.0$ & $46.4 \pm 0.6$ \\
\hline $\begin{array}{l}\text { Ponderal index } \\
\quad\left(\mathrm{g} \cdot \mathrm{cm}^{-3} \cdot 10^{3}\right)\end{array}$ & $43.1 \pm 2.2$ & $41.8 \pm 1.2$ & $45.9 \pm 2.4$ & $43.6 \pm 1.7$ \\
\hline Hindlimb length $(\mathrm{cm})$ & $33.7 \pm 0.5^{\mathrm{a}}$ & $31.3 \pm 0.8^{\mathrm{b}}$ & $33.4 \pm 0.5^{\mathrm{ab}}$ & $33.5 \pm 0.5^{\mathrm{ab}}$ \\
\hline Brain weight $(\mathrm{g})$ & $45.6 \pm 1.5$ & $46.9 \pm 2.1$ & $48.3 \pm 1.3$ & $47.8 \pm 1.3$ \\
\hline Liver weight $(\mathrm{g})$ & $149 \pm 7$ & $144 \pm 5$ & $140 \pm 5$ & $167 \pm 19$ \\
\hline Kidney weight (g) & $15.2 \pm 1.1$ & $13.8 \pm 0.5$ & $16.3 \pm 2.0$ & $16.9 \pm 1.6$ \\
\hline Adrenal weight $(\mathrm{g})$ & $0.27 \pm 0.02$ & $0.25 \pm 0.04$ & $0.26 \pm 0.02$ & $0.28 \pm 0.03$ \\
\hline Spleen weight $(\mathrm{g})$ & $10.5 \pm 1.6$ & $8.7 \pm 0.6$ & $9.3 \pm 0.5$ & $12.9 \pm 2.4$ \\
\hline Heart weight $(\mathrm{g})$ & $34.8 \pm 1.8^{\mathrm{A}}$ & $29.3 \pm 1.5^{\mathrm{B}}$ & $34.8 \pm 1.7^{\mathrm{A}}$ & $33.3 \pm 0.9^{\mathrm{AB}}$ \\
\hline Lung weight $(\mathrm{g})$ & $119 \pm 4$ & $114 \pm 4$ & $127 \pm 5$ & $120 \pm 8$ \\
\hline Chest thymus weight (g) & $5.9 \pm 0.8$ & $5.3 \pm 0.6$ & $5.7 \pm 1.0$ & $4.8 \pm 0.4$ \\
\hline Neck thymus weight $(\mathrm{g})$ & $11.2 \pm 1.1$ & $10.1 \pm 0.6$ & $11.6 \pm 1.5$ & $9.2 \pm 0.5$ \\
\hline Thyroid weight $(\mathrm{g})$ & $1.20 \pm 0.10$ & $0.93 \pm 0.09$ & $1.03 \pm 0.10$ & $1.02 \pm 0.07$ \\
\hline Pancreas weight (g) & $3.3 \pm 0.2$ & $3.1 \pm 0.2$ & $4.1 \pm 0.3$ & $3.7 \pm 0.3$ \\
\hline Membranes weight (g) & $311 \pm 18$ & $424 \pm 51$ & $385 \pm 59$ & $326 \pm 44$ \\
\hline Amniotic fluid weight $(\mathrm{g})$ & $980 \pm 134$ & $1286 \pm 348$ & $949 \pm 162$ & $1356 \pm 261$ \\
\hline Placentome number & $81.6 \pm 3.3$ & $81.5 \pm 5.0$ & $83.5 \pm 5.9$ & $78.0 \pm 7.4$ \\
\hline Placenta weight $(\mathrm{g})$ & $536 \pm 29$ & $628 \pm 25$ & $542 \pm 15$ & $601 \pm 41$ \\
\hline$\%$ A placentomes by weight & $22 \pm 8$ & $25 \pm 4$ & $18 \pm 7$ & $11 \pm 3$ \\
\hline$\%$ B placentomes by weight & $49 \pm 9$ & $36 \pm 6$ & $57 \pm 11$ & $41 \pm 9$ \\
\hline$\% \mathrm{C}$ placentomes by weight & $16 \pm 5$ & $22 \pm 6$ & $14 \pm 5$ & $22 \pm 5$ \\
\hline$\%$ D placentomes by weight & $14 \pm 9$ & $17 \pm 6$ & $11 \pm 9$ & $26 \pm 10$ \\
\hline Fetal: placental weight ratio & $8.4 \pm 0.5^{\mathrm{a}}$ & $6.2 \pm 0.3^{\mathrm{b}}$ & $8.2 \pm 0.4^{\mathrm{a}}$ & $7.4 \pm 0.4^{\mathrm{ab}}$ \\
\hline
\end{tabular}

Data are mean \pm SEM. Different uppercase letters $p<0.10$ and lowercase letters $p<0.05$ for comparisons between groups within the same time period. There were no significant differences between groups in organ weights as a percentage of body weight.

Pre-C, preconception; Post-C, postconception.

Table 3. Relationship between maternal weight changes before and after mating and fetal measurements at $132 d$

\begin{tabular}{|c|c|c|c|c|}
\hline Fetal variable & Maternal variable & Correlation coefficient & $t$ ratio & $p$ value \\
\hline \multirow[t]{3}{*}{ Weight (kg) } & Weight change before mating & $2.9 \pm 13.8 \times 10^{-3}$ & 0.21 & 0.84 \\
\hline & Weight change after mating & $-27.0 \pm 16.6 \times 10^{-3}$ & -1.63 & 0.11 \\
\hline & Interaction & $4.7 \pm 1.8 \times 10^{-3}$ & 2.66 & 0.01 \\
\hline \multirow[t]{3}{*}{ Chest girth $(\mathrm{cm})$} & Weight change before mating & $-0.07 \pm 0.06$ & -1.26 & 0.22 \\
\hline & Weight change after mating & $-0.14 \pm 0.07$ & -2.05 & 0.05 \\
\hline & Interaction & $0.016 \pm 0.007$ & 2.25 & 0.03 \\
\hline \multirow[t]{3}{*}{ Hindlimb length $(\mathrm{cm})$} & Weight change before mating & $0.025 \pm 0.050$ & 0.50 & 0.62 \\
\hline & Weight change after mating & $-0.084 \pm 0.060$ & -1.38 & 0.18 \\
\hline & Interaction & $0.017 \pm 0.006$ & 2.79 & $<0.01$ \\
\hline
\end{tabular}

Fetal weight $\left(r^{2}=0.21, F=2.6, p=0.07\right)$, chest girth $\left(r^{2}=0.18, F=2.1, p=0.12\right)$, hindlimb length $\left(r^{2}=0.23, F=2.9, p=0.05\right)$, and placenta weight $\left(r^{2}=0.26, F=3.6, p=0.02\right)$. 
$56 \%$ with maternal fasting $(2.90 \pm 0.16$ to $1.27 \pm 0.38$ $\mathrm{mm} \cdot \mathrm{d}^{-1}, p=0.02$; Fig. 3). After refeeding, their growth rate was slower than before fasting $\left(2.72 \pm 0.20 \mathrm{~mm} \cdot \mathrm{d}^{-1}, p=\right.$ 0.01 ) and similar to controls. At postmortem, they were in similar size to controls (Table 2).

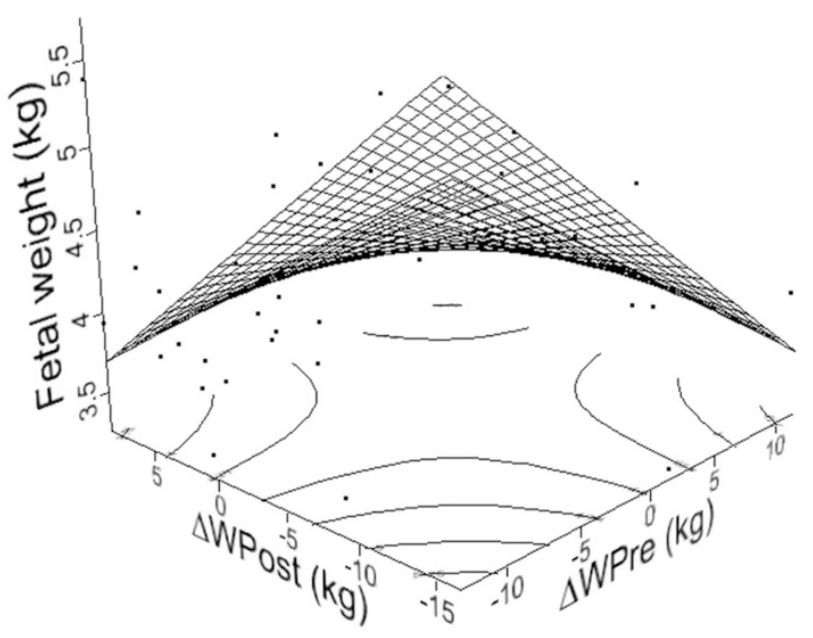

Figure 4. The interaction of maternal preconception weight change ( -70 to $-2 \mathrm{~d}, \Delta \mathrm{WPre}$ ) and postconception weight change ( 0 to $30 \mathrm{~d}, \Delta$ WPost) on fetal weight at $132 \mathrm{~d}(p=0.01)$.
Effects of maternal weight parameters on fetal growth. There were no independent effects of ewe weight at $-71 \mathrm{~d}$, or change in ewe weight before $(-70$ to $-2 \mathrm{~d})$ or after mating $(0$ to $30 \mathrm{~d}$ ) on fetal size at postmortem (Table 3 ). However, there was an interaction between weight change before and after mating (Fig. 4). Ewes that gained most weight both before and after mating had the largest fetuses, whereas ewes that gained weight before and lost weight after mating, or vice versa, had the smallest fetuses. This pattern was similar for fetal weight, chest girth, and hind limb length (Table 3).

Metabolism. Control ewes maintained stable plasma glucose, urea, insulin, and IGF-1 concentrations from $-61 \mathrm{~d}$ to $30 \mathrm{~d}$ (Table 4). Plasma glucose concentration remained stable after this, but insulin, IGF-1, and $\beta$ HBA concentrations increased, whereas urea concentrations decreased. Plasma FFA concentrations were higher at 45 to $97 \mathrm{~d}$, but decreased again by 114 to $121 \mathrm{~d}$ (Table 4).

Maternal plasma glucose, urea, and insulin concentrations fell during undernutrition in all undernourished groups, but plasma IGF-1 concentrations did not change (Table 4). $\beta$ HBA and FFA concentrations rose during undernutrition only in the pre-C group. By late gestation, concentrations of all metabolites and hormones were similar among groups, except that plasma insulin concentrations were higher in the post- $\mathrm{C}$ than

Table 4. Maternal and fetal plasma metabolite and hormone concentrations

\begin{tabular}{|c|c|c|c|c|c|c|c|c|}
\hline & \multicolumn{6}{|c|}{ Maternal } & \multicolumn{2}{|c|}{ Fetal } \\
\hline & $-61 \mathrm{~d}$ & -60 to $0 \mathrm{~d}$ & 0 to $30 \mathrm{~d}$ & 45 to $97 \mathrm{~d}$ & 114 to $121 \mathrm{~d}$ & 128 to $131 \mathrm{~d}$ & 114 to $121 \mathrm{~d}$ & 128 to $131 \mathrm{~d}$ \\
\hline \multicolumn{9}{|c|}{ Glucose $\left(\mathrm{mmol} \cdot \mathrm{L}^{-1}\right)$} \\
\hline Control & $3.99 \pm 0.13$ & $3.70 \pm 0.10^{\mathrm{AB}}$ & $3.60 \pm 0.07$ & $3.73 \pm 0.12$ & $3.67 \pm 0.06$ & $3.69 \pm 0.08$ & $1.02 \pm 0.04^{\mathrm{AB}}$ & $1.03 \pm 0.07$ \\
\hline Pre-C & $3.76 \pm 0.10$ & $3.38 \pm 0.04 * * \mathrm{~B}$ & $3.62 \pm 0.11^{* *}$ & $3.51 \pm 0.08$ & $3.66 \pm 0.07 *$ & $3.53 \pm 0.07$ & $1.08 \pm 0.05^{\mathrm{A}}$ & $1.02 \pm 0.09$ \\
\hline Post-C & $3.84 \pm 0.17$ & $3.77 \pm 0.14^{\mathrm{A}}$ & $3.43 \pm 0.10^{* *}$ & $3.64 \pm 0.12^{* *}$ & $3.66 \pm 0.09$ & $3.63 \pm 0.08$ & $1.05 \pm 0.05^{\mathrm{AB}}$ & $0.98 \pm 0.03^{*}$ \\
\hline Pre + post-C & $4.08 \pm 0.26$ & $3.49 \pm 0.08 * \mathrm{AB}$ & $3.42 \pm 0.07$ & $3.52 \pm 0.06$ & $3.53 \pm 0.05$ & $3.52 \pm 0.04$ & $0.91 \pm 0.05^{\mathrm{B}}$ & $0.93 \pm 0.06$ \\
\hline \multicolumn{9}{|c|}{ Insulin $\left(\mathrm{ng} \cdot \mathrm{mL}^{-1}\right)$} \\
\hline Control & $0.17 \pm 0.03$ & $0.18 \pm 0.02$ & $0.17 \pm 0.02$ & $0.18 \pm 0.03^{\mathrm{A}}$ & $0.32 \pm 0.03^{* \mathrm{ab}}$ & $0.44 \pm 0.05^{* *}$ & $0.19 \pm 0.02$ & $0.23 \pm 0.03$ \\
\hline Pre-C & $0.21 \pm 0.03$ & $0.14 \pm 0.01 *$ & $0.15 \pm 0.02$ & $0.16 \pm 0.02^{\mathrm{AB}}$ & $0.34 \pm 0.04 * \mathrm{ab}$ & $0.34 \pm 0.04$ & $0.20 \pm 0.02$ & $0.22 \pm 0.03$ \\
\hline Post-C & $0.18 \pm 0.02$ & $0.20 \pm 0.05$ & $0.12 \pm 0.03 *$ & $0.18 \pm 0.03 * \mathrm{AB}$ & $0.44 \pm 0.05^{* * a}$ & $0.34 \pm 0.04 * *$ & $0.21 \pm 0.03$ & $0.25 \pm 0.03$ \\
\hline Pre + post $-\mathrm{C}$ & $0.19 \pm 0.05$ & $0.13 \pm 0.02$ & $0.13 \pm 0.02$ & $0.11 \pm 0.02^{\mathrm{B}}$ & $0.25 \pm 0.02 * * \mathrm{~b}$ & $0.35 \pm 0.04 *$ & $0.21 \pm 0.02$ & $0.22 \pm 0.03$ \\
\hline \multicolumn{9}{|c|}{ IGF-1 $\left(\mathrm{ng} \cdot \mathrm{mL}^{-1}\right)$} \\
\hline Control & $43.5 \pm 3.1$ & $40.8 \pm 1.8$ & $45.6 \pm 2.4$ & $46.5 \pm 2.1$ & $69.7 \pm 9.8 *$ & $91.8 \pm 14.0^{* *}$ & $70.4 \pm 5.8$ & $71.2 \pm 5.2$ \\
\hline Pre-C & $39.9 \pm 2.7$ & $44.9 \pm 2.3$ & $46.7 \pm 1.6$ & $45.9 \pm 1.6$ & $53.3 \pm 5.3$ & $58.1 \pm 5.3$ & $55.9 \pm 4.2$ & $54.4 \pm 9.7$ \\
\hline Post-C & $42.1 \pm 5.9$ & $42.9 \pm 3.8$ & $41.3 \pm 1.3$ & $47.4 \pm 1.9$ & $61.0 \pm 9.6$ & $86.3 \pm 14.8^{*}$ & $76.4 \pm 9.4$ & $94.4 \pm 13.2^{*}$ \\
\hline Pre + post-C & $41.7 \pm 2.4$ & $40.8 \pm 1.9$ & $41.8 \pm 2.1$ & $44.4 \pm 2.0$ & $46.6 \pm 5.0$ & $64.7 \pm 56.9^{* *}$ & $62.4 \pm 6.1$ & $77.5 \pm 13.2$ \\
\hline \multicolumn{9}{|c|}{$\beta \mathrm{HBA}\left(\mathrm{mmol} \cdot \mathrm{L}^{-1}\right)$} \\
\hline Control & $0.14 \pm 0.01$ & $0.20 \pm 0.01 * *$ & $0.19 \pm 0.01^{\mathrm{b}}$ & $0.24 \pm 0.02 *$ & $0.45 \pm 0.02 * * \mathrm{AB}$ & $0.49 \pm 0.03$ & - & - \\
\hline Pre-C & $0.18 \pm 0.04$ & $0.19 \pm 0.01$ & $0.20 \pm 0.01^{\mathrm{b}}$ & $0.20 \pm 0.02$ & $0.41 \pm 0.05 * * \mathrm{~B}$ & $0.47 \pm 0.05$ & - & - \\
\hline Post-C & $0.20 \pm 0.09$ & $0.19 \pm 0.02$ & $0.39 \pm 0.03^{* * a}$ & $0.23 \pm 0.02 * *$ & $0.55 \pm 0.05 * * \mathrm{~A}$ & $0.36 \pm 0.04^{*}$ & - & - \\
\hline Pre + post-C & $0.18 \pm 0.02$ & $0.20 \pm 0.01$ & $0.21 \pm 0.01^{\mathrm{b}}$ & $0.21 \pm 0.01$ & $0.44 \pm 0.04 * * \mathrm{AB}$ & $0.42 \pm 0.03$ & - & - \\
\hline \multicolumn{9}{|c|}{$\mathrm{FFA}\left(\mathrm{mmol} \cdot \mathrm{L}^{-1}\right)$} \\
\hline Control & $0.52 \pm 0.07$ & $0.35 \pm 0.04 * \mathrm{~b}$ & $0.31 \pm 0.06^{\mathrm{b}}$ & $0.58 \pm 0.07 * *$ & $0.22 \pm 0.03 * *$ & $0.15 \pm 0.04$ & - & - \\
\hline Pre-C & $0.38 \pm 0.10$ & $0.53 \pm 0.04^{\mathrm{ab}}$ & $0.25 \pm 0.07 * \mathrm{~b}$ & $0.35 \pm 0.06$ & $0.20 \pm 0.04 * *$ & $0.20 \pm 0.06$ & - & - \\
\hline Post-C & $0.42 \pm 0.09$ & $0.37 \pm 0.07^{\mathrm{ab}}$ & $1.23 \pm 0.07 * * \mathrm{a}$ & $0.43 \pm 0.08 * *$ & $0.22 \pm 0.06^{*}$ & $0.26 \pm 0.04$ & - & - \\
\hline Pre + post-C & $0.58 \pm 0.12$ & $0.56 \pm 0.09^{\mathrm{a}}$ & $0.51 \pm 0.07^{\mathrm{b}}$ & $0.40 \pm 0.07 *$ & $0.19 \pm 0.03 * *$ & $0.17 \pm 0.03$ & - & - \\
\hline \multicolumn{9}{|c|}{ Urea $\left(\mathrm{mmol} \cdot \mathrm{L}^{-1}\right)$} \\
\hline Control & $6.54 \pm 0.44$ & $7.00 \pm 0.27^{\mathrm{a}}$ & $7.32 \pm 0.20^{\mathrm{a}}$ & $5.92 \pm 0.18^{* *}$ & $5.03 \pm 0.28 * *$ & $4.18 \pm 0.30^{*}$ & $5.65 \pm 0.25$ & $4.81 \pm 0.23^{* *}$ \\
\hline Pre-C & $6.28 \pm 0.57$ & $5.40 \pm 0.28^{\mathrm{b}}$ & $6.05 \pm 0.24 * \mathrm{~b}$ & $5.73 \pm 0.17$ & $5.13 \pm 0.28$ & $3.91 \pm 0.53^{*}$ & $5.65 \pm 0.26$ & $4.53 \pm 0.42$ \\
\hline Post-C & $5.50 \pm 0.44$ & $6.97 \pm 0.57^{\mathrm{a}}$ & $4.75 \pm 0.25^{* \mathrm{c}}$ & $5.40 \pm 0.40^{*}$ & $4.33 \pm 0.61 *$ & $3.92 \pm 0.71$ & $5.21 \pm 0.62$ & $4.50 \pm 0.67$ \\
\hline Pre + post-C & $5.78 \pm 0.46$ & $5.35 \pm 0.27^{\mathrm{b}}$ & $5.52 \pm 0.23^{\mathrm{bc}}$ & $5.63 \pm 0.20$ & $5.13 \pm 0.38$ & $4.11 \pm 0.31 *$ & $5.76 \pm 0.35$ & $4.62 \pm 0.21 * *$ \\
\hline
\end{tabular}

Data are mean \pm SEM. ${ }^{*} p<0.05, * * p<0.01$ for comparisons with previous time period within the same group. Different uppercase letters $p<0.10$ and different lowercase letters $p<0.05$ for comparisons between groups within the same time period.

Pre-C, preconception; Post-C, postconception. 

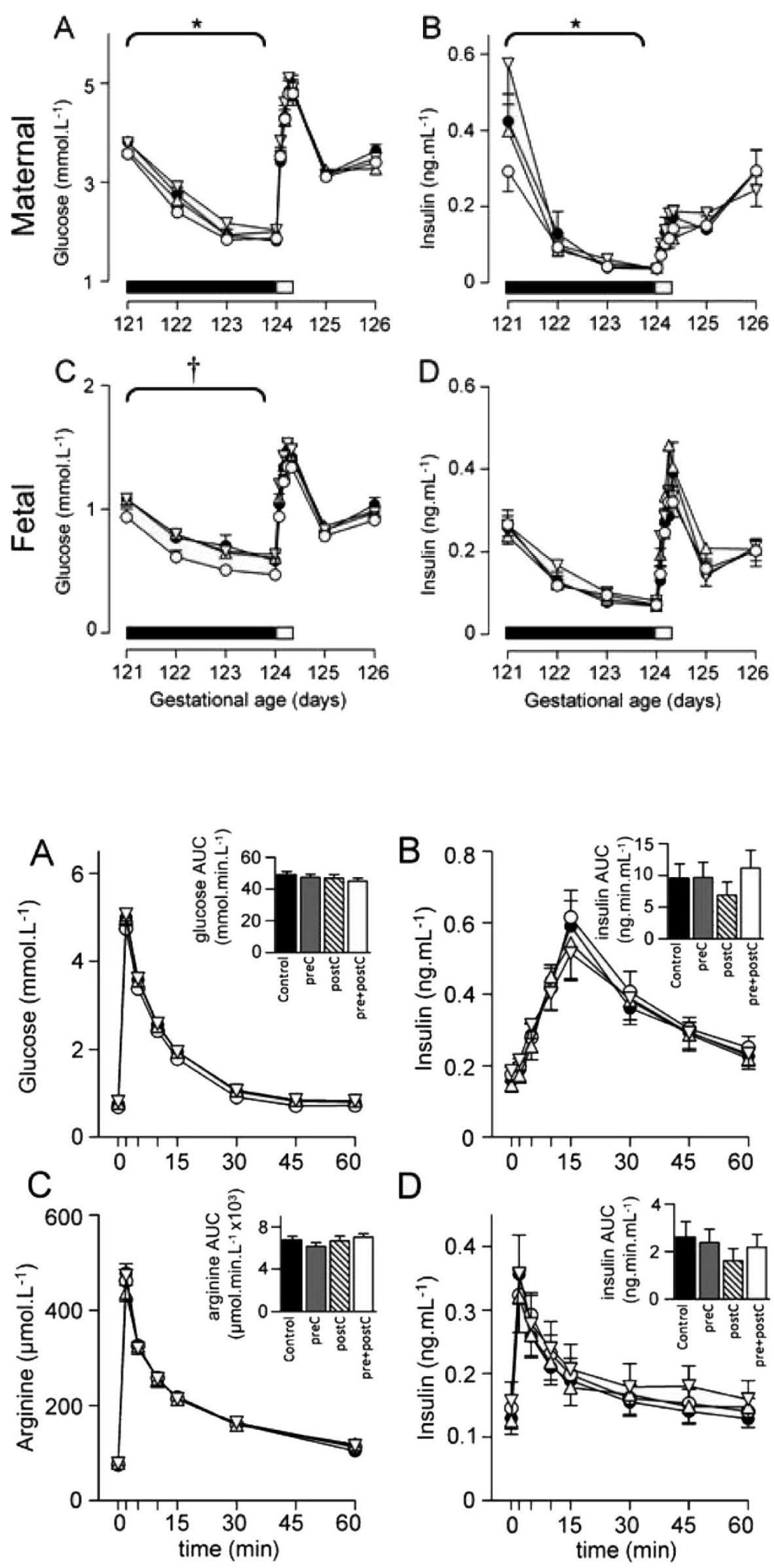

Figure 5. Maternal plasma glucose $(A)$ and insulin $(B)$, and fetal plasma glucose $(C)$ and insulin $(D)$ concentrations during fasting (black bar) and refeeding (open bar). Control $\bigcirc(n=$ $10)$, pre-C $\triangle(n=10)$, post-C $\nabla(n=9)$, and pre + post-C $\bigcirc(n=12)$. Nutrition effect: ${ }^{*} p<$ 0.05 for pre + post-C $v s$ post-C, $\dagger p<0.01$ for pre + post-C $v s$ all other groups.
Figure 6. Fetal glucose $(A)$ and insulin $(B)$ responses to an i.v. glucose tolerance test; fetal arginine $(C)$ and insulin $(D)$ responses to an i.v. arginine challenge. Area under the curves shown as inset histograms. Control $\bullet(n=16)$, pre-C $\triangle(n=12)$, post-C $\nabla(n=11)$, pre + post-C $\bigcirc$ $(n=13)$. There were no significant differences between groups. 
pre + post-C group at 114 to $121 \mathrm{~d}$. Fetal plasma urea, insulin, IGF-I (Table 4), and lactate concentrations were similar among groups in late gestation. Fetal plasma glucose concentrations tended to be lower in the pre + post-C group $(p=0.07)$.

Maternal plasma glucose, insulin (Fig. 5), and IGF-I concentrations $\left(80.7 \pm 5.3\right.$ to $\left.44.2 \pm 3.4 \mathrm{ng} \cdot \mathrm{mL}^{-1}, p<0.01\right)$ decreased with fasting in all groups, whereas urea $(4.8 \pm 0.3$ to $\left.7.5 \pm 0.3 \mathrm{mmol} \cdot \mathrm{L}^{-1}, p<0.01\right)$, FFA $(0.16 \pm 0.03$ to $\left.1.96 \pm 0.05 \mathrm{mmol} \cdot \mathrm{L}^{-1}, p<0.01\right)$, and $\beta \mathrm{HBA}(0.52 \pm 0.03$ to $\left.1.62 \pm 0.08 \mathrm{mmol} \cdot \mathrm{L}^{-1}, p<0.01\right)$ concentrations increased. Plasma glucose and insulin were lower in pre + post- $C$ than post-C ewes during fasting, but there were no other differences in metabolite and hormone concentrations among groups.

Fetal plasma glucose, insulin (Fig. 5), and IGF-I concentrations $\left(74.0 \pm 3.7\right.$ to $\left.53.2 \pm 6.4 \mathrm{ng} \cdot \mathrm{mL}^{-1}, p<0.01\right)$ decreased with fasting in all groups, whereas urea concentrations increased (5.5 \pm 0.3 to $\left.8.7 \pm 0.3 \mathrm{mmol} \cdot \mathrm{L}^{-1}, p<0.01\right)$. Plasma glucose concentrations were lower in pre + post-C fetuses during fasting than in other groups $(p<0.01)$, but there were no other differences in metabolite and hormone concentrations among groups.

Fetal glucose and arginine challenges. Nutritional group had no effect on AUC for glucose and insulin after the glucose bolus, or on peak glucose or insulin concentrations (Fig. 6). Nutritional group had no effect on AUC for arginine and insulin after the arginine bolus, or on peak plasma arginine or insulin concentrations (Fig. 6).

Maternal HPAA. Nutritional group had no effect on maternal plasma ACTH, cortisol, cortisone or progesterone concentrations, or on cortisol:cortisone and cortisol:ACTH ratios (Table 5).

Fetal HPAA. Nutritional group had no effect on baseline fetal plasma concentrations of ACTH, cortisol, cortisone and DHEA, or on cortisol:cortisone and cortisol:ACTH ratios (Table 5). Fetal plasma cortisol concentrations and cortisol:cortisone and cortisol:ACTH ratios increased from $114-121 \mathrm{~d}$ to $128-131 \mathrm{~d}$ in all groups.

Nutritional group had no effect on AUC for ACTH or cortisol, or cortisol AUC:ACTH AUC ratios in response to the $\mathrm{CRH}$ and AVP challenge (Fig. 7).

Metyrapone reduced mean fetal plasma cortisol concentrations to $1.5 \pm 0.3 \mathrm{ng} \cdot \mathrm{mL}^{-1}$ at $30 \min (p<0.01)$, with no difference among groups (Fig. 7). Nutritional group had no effect on ACTH AUC, 11-deoxycortisol AUC, or 11deoxycortisol AUC:ACTH AUC ratios.

There were no effects of fetal sex on any of the parameters measured.

\section{DISCUSSION}

This study aimed to distinguish between the effects of preconception and postconception undernutrition on fetal growth, metabolism, and endocrinology in late gestation. We have previously shown that undernutrition from $60 \mathrm{~d}$ before to $30 \mathrm{~d}$ after conception affects the growth $(6,7)$, glucose-insulin axis (8), and HPAA (9) of late gestation fetal sheep. We hypothesized that these effects would differ if undernutrition
Table 5. Maternal and fetal plasma ACTH and steroid concentrations

\begin{tabular}{|c|c|c|c|c|}
\hline & \multicolumn{2}{|c|}{ Maternal } & \multicolumn{2}{|c|}{ Fetal } \\
\hline & 114 to $121 \mathrm{~d}$ & 128 to $131 \mathrm{~d}$ & 114 to $121 \mathrm{~d}$ & 128 to $131 \mathrm{~d}$ \\
\hline \multicolumn{5}{|c|}{$\mathrm{ACTH}\left(\mathrm{pg} \cdot \mathrm{mL}^{-1}\right)$} \\
\hline Control & $33.2 \pm 5.8$ & $33.9 \pm 10.8$ & $20.7 \pm 4.7$ & $19.6 \pm 2.5$ \\
\hline Pre-C & $26.8 \pm 3.2$ & $31.8 \pm 1.8$ & $20.9 \pm 2.4$ & $30.4 \pm 7.8$ \\
\hline Post-C & $37.8 \pm 5.9$ & $36.9 \pm 8.0$ & $18.6 \pm 2.5$ & $21.7 \pm 4.8$ \\
\hline Pre + post-C & $25.7 \pm 13.4$ & $34.1 \pm 3.1^{*}$ & $17.2 \pm 1.1$ & $15.8 \pm 2.0$ \\
\hline \multicolumn{5}{|c|}{ Cortisol $\left(\mathrm{ng} \cdot \mathrm{mL}^{-1}\right)$} \\
\hline Control & $9.4 \pm 2.4$ & $4.3 \pm 0.9$ & $0.96 \pm 0.31$ & $4.94 \pm 1.89 *$ \\
\hline Pre-C & $6.7 \pm 1.3$ & $2.4 \pm 0.4 *$ & $1.30 \pm 0.77$ & $4.98 \pm 1.88^{*}$ \\
\hline Post-C & $7.9 \pm 1.5$ & $7.2 \pm 1.2$ & $0.73 \pm 0.17$ & $2.33 \pm 0.54 * *$ \\
\hline Pre + post-C & $7.1 \pm 1.6$ & $5.6 \pm 2.6$ & $1.00 \pm 0.20$ & $3.44 \pm 1.05^{*}$ \\
\hline \multicolumn{5}{|c|}{ Cortisone $\left(\mathrm{ng} \cdot \mathrm{mL}^{-1}\right)$} \\
\hline Control & $2.1 \pm 0.2$ & $1.7 \pm 0.1$ & $1.37 \pm 0.39$ & $3.50 \pm 1.51$ \\
\hline Pre-C & $1.6 \pm 0.1$ & $1.3 \pm 0.2$ & $1.26 \pm 0.25$ & $3.65 \pm 1.47$ \\
\hline Post-C & $2.1 \pm 0.3$ & $2.1 \pm 0.2$ & $1.29 \pm 0.27$ & $2.09 \pm 0.40 *$ \\
\hline Pre + post-C & $1.6 \pm 0.2$ & $1.6 \pm 0.5$ & $1.03 \pm 0.20$ & $1.54 \pm 0.21$ \\
\hline \multicolumn{5}{|c|}{ Cortisol:ACTH ratio } \\
\hline Control & $258 \pm 86$ & $143 \pm 60$ & $39 \pm 11$ & $156 \pm 52 *$ \\
\hline Pre-C & $237 \pm 70$ & $67 \pm 14^{*}$ & $31 \pm 11$ & $154 \pm 27 * *$ \\
\hline Post-C & $192 \pm 66$ & $204 \pm 66$ & $33 \pm 8$ & $107 \pm 40 * *$ \\
\hline Pre + post-C & $224 \pm 75$ & $94 \pm 41 *$ & $47 \pm 10$ & $151 \pm 44 * *$ \\
\hline \multicolumn{5}{|c|}{ Cortisol:cortisone ratio } \\
\hline Control & $3.7 \pm 0.5$ & $3.1 \pm 0.6$ & $0.74 \pm 0.07$ & $1.82 \pm 0.35^{*}$ \\
\hline Pre-C & $3.8 \pm 0.5$ & $1.9 \pm 0.2^{*}$ & $0.67 \pm 0.17$ & $1.22 \pm 0.25^{*}$ \\
\hline Post-C & $3.5 \pm 0.6$ & $3.4 \pm 0.6$ & $0.56 \pm 0.07$ & $1.63 \pm 0.30^{* *}$ \\
\hline $\mathrm{Pre}+$ post-C & $3.8 \pm 0.5$ & $2.6 \pm 0.4^{*}$ & $0.97 \pm 0.16$ & $1.76 \pm 0.36^{*}$ \\
\hline \multicolumn{5}{|c|}{$\begin{array}{l}\text { Maternal progesterone }\left(\mathrm{ng} \cdot \mathrm{mL}^{-1}\right) \\
\text { or }\end{array}$} \\
\hline \multicolumn{5}{|c|}{ Fetal DHEA $\left(\mathrm{ng} \cdot \mathrm{mL}^{-1}\right)$} \\
\hline Control & $9.0 \pm 0.4$ & $9.0 \pm 0.7$ & $0.76 \pm 0.13$ & $0.73 \pm 0.18$ \\
\hline Pre-C & $8.7 \pm 0.8$ & $10.6 \pm 1.3$ & $0.55 \pm 0.05$ & $0.43 \pm 0.04 *$ \\
\hline Post-C & $10.4 \pm 1.2$ & $10.7 \pm 1.0$ & $0.76 \pm 0.15$ & $0.64 \pm 0.11$ \\
\hline Pre + post $-\mathrm{C}$ & $10.0 \pm 0.6$ & $10.5 \pm 1.2$ & $0.53 \pm 0.08$ & $0.62 \pm 0.10$ \\
\hline
\end{tabular}

Data are mean \pm SEM. There were no significant differences between nutritional groups. $* p<0.05$, $* * p<0.01$ for comparison with previous time period within the same group.

Pre-C, preconception; Post-C, postconception.

was restricted to the preconception or postconception period alone.

Fetal Growth. Preconception and postconception undernutrition affected fetal growth in late gestation in different ways. In ewes exposed to undernutrition only before conception, growth of fetuses in late gestation was more sensitive to the stressors of surgery (which includes a 24-h fast) and a later $3 \mathrm{~d}$ maternal fast. Pre-C fetuses grew more slowly after surgery and dramatically slowed growth in response to the $3 \mathrm{~d}$ maternal fast. These fetuses were smaller at postmortem at $132 \mathrm{~d}$ and had lower fetal:placental weight ratios. Undernutrition both before and after conception did not reduce fetal growth rate following surgery, but did result in a significant slowing of growth in response to the $3 \mathrm{~d}$ maternal fast. These fetuses were of a similar size to controls at postmortem. Undernutrition only after conception resulted in fetuses that tended to be smaller at $110 \mathrm{~d}$ but grew faster in late gestation and were of similar size to controls at postmortem. Neither this group nor controls reduced their growth rate in response to the $3 \mathrm{~d}$ maternal fast.

These data suggest that maternal nutrition around the time of conception determines, at least in part, fetal growth re- 


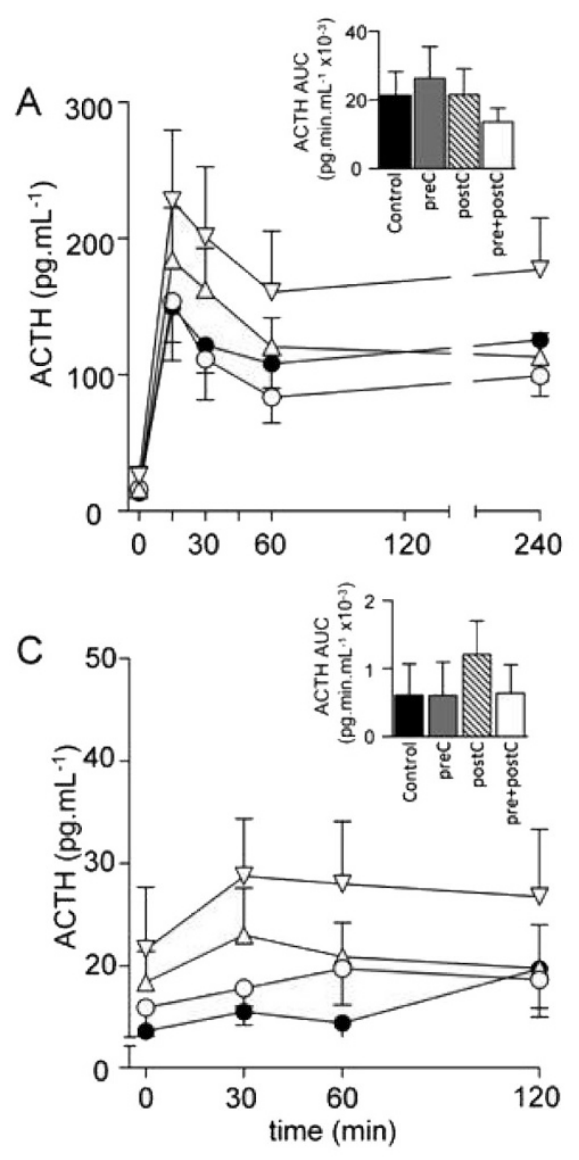

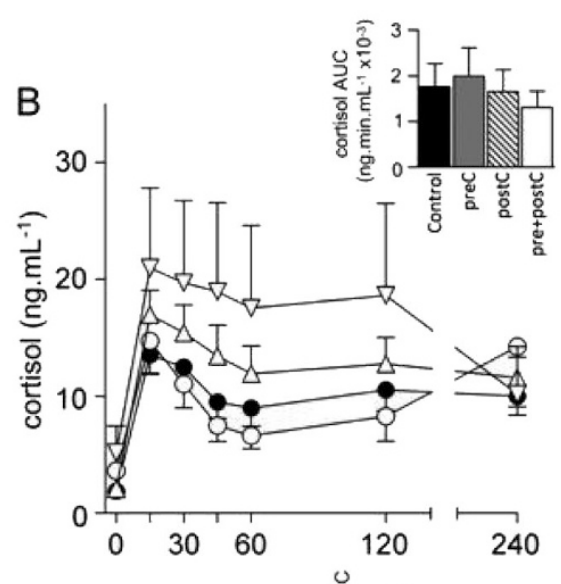

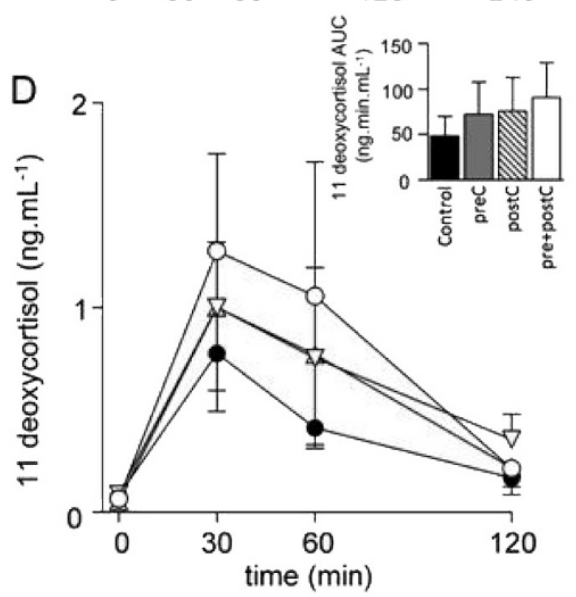

Figure 7. Fetal ACTH $(A)$ and cortisol $(B)$ responses to CRH+AVP challenge, and to metyrapone challenge $(C$ and $D)$. Area under the curves shown as inset histograms. Control $(n=9)$, pre-C $\triangle(n=9)$, post-C $\nabla(n=8)$, pre + post-C $\bigcirc(n=11)$. There were no significant differences between groups.

sponses to adverse events later in pregnancy. The preconceptional period seems to be a particularly important period in this regard. Should similar interactions occur in human pregnancy, then clearly this would have important clinical implications. This study also reinforces the concept that offspring of similar sizes may result from different prenatal growth trajectories $(6,15)$.

The finding that smallest fetuses were from ewes that gained weight before mating and lost weight after mating, or vice versa, suggests an interaction between nutrition in these two periods. The similar growth rate of pre + post-C and control fetuses is further evidence of this interaction. Changes in maternal nutrition may result in altered plasma metabolite and hormone concentrations, which in turn may alter the oviductal and uterine environment of early embryos. For example, the onset of and relief from undernutrition in sheep produces acute changes in plasma glucocorticoid concentrations (21). In women, low preconception BMI is associated with low birth weight (22), similar to our pre-C group, and the pattern of weight gain during pregnancy influences birth weight (23).

Our finding that controls did not significantly slow their growth with maternal fasting, but that both pre+post-C and pre-C fetuses did, contrasts with previous reports that fetuses of periconceptionally undernourished ewes, but not controls, maintained their slow growth rate during undernutrition (7). However, the duration, severity, and timing of the nutritional insult (48 h fast at 121 versus $10 \mathrm{~d}$ undernutrition at $105 \mathrm{~d}$ ) might have influenced the fetal growth response. Similarly, we did not find that periconceptional undernutrition resulted in the slower fetal growth previously reported in the period before parturition (126 to 145 d) (6), perhaps because our study was too early to detect such changes.

Maternal changes. Inadequate blood volume expansion (24), reduced uterine blood flow (25), and poor maternal weight gain (23) are associated with human fetal growth restriction. We found pre+post-C ewes were still lighter in late gestation, had greater food intake, but lower plasma glucose and insulin concentrations, especially during the fast. Fetal plasma glucose concentrations reflected the low maternal concentrations and fell further during the fast, which may account for their slower growth.

The increased food intake of these ewes may reflect an attempt to recover body weight and provide for the fetus, but fasting exposed their lack of nutritional reserves. Nutrient partitioning in a weight gain phase, as in adolescent pregnancy, seems to favor the ewe over the placenta and fetus (26). Periconceptional undernutrition also disturbs the normal development of maternal insulin resistance (27) The lower fetal glucose concentrations are unlikely to reflect inadequate uterine circulation, as periconceptionally undernourished sheep have normal blood volume and increased uterine blood flow in late gestation (28).

Although altered maternal nutrition can increase circulating glucocorticoid concentrations (29), there are few data on the effect of periconceptional undernutrition on maternal HPAA. 
We found no differences between groups in maternal plasma glucocorticoid concentrations in late gestation, suggesting that if fetal exposure to glucocorticoids contributes to differences in growth and metabolism, the mechanism must lie in the placenta or fetus rather than maternal HPAA. Chronic nutrient restriction from $26 \mathrm{~d}$ in sheep reduces placental $11 \beta$-hydroxysteroid dehydrogenase type 2 activity in late gestation, but there is no effect if the nutritional restriction is limited to early, mid or late gestation (30). Placental $11 \beta$-hydroxysteroid dehydrogenase type 2 activity was also reduced and cortisol:cortisone ratio increased at $85 \mathrm{~d}$ after periconceptional undernutrition (31).

Placental changes. The pre- $\mathrm{C}$ group had larger placenta and smaller fetuses, with a smaller fetal:placental weight ratio. This pattern has been reported after maternal undernutrition in midgestation (32), but our data suggest that maternal nutritional status before mating can also influence subsequent fetoplacental growth. These fetuses also grew more slowly than controls; a difference accentuated with maternal fasting. It is possible that the larger placenta inhibited fetal growth by using a greater proportion of nutrients, even though plasma glucose and insulin concentrations were maintained. It is also possible that placental transfer capacity was decreased, leading to slower fetal growth.

Fetal changes. We have previously shown that periconceptional undernutrition similar to the pre + post-C group increased fetal insulin response to a glucose challenge (8) and increased response to metyrapone (9), suggesting accelerated fetal pancreatic and HPAA maturation, consistent with the tendency to preterm delivery (33). However, no such changes were found here, for several possible reasons. First, the dose of metyrapone was higher than in earlier studies, increasing the stimulus to ACTH production and possibly obscuring differences seen at lower doses. Second, this study occurred over 3 years, so flock or seasonal differences may have increased data variability. Third, the mean weight of controls increased from 64 to $68 \mathrm{~kg}$ during this study, compared with 56 to $70 \mathrm{~kg}$ in earlier studies. It is possible that the lower starting weight and greater weight change in the earlier studies was critical. Weight change in the periconceptional period relates to fetoplacental growth in early pregnancy (11), and in women weight gain relates to the risk of preterm delivery (34). Undernutrition from $60 \mathrm{~d}$ before to $7 \mathrm{~d}$ after mating also had no effect on ACTH concentrations in late gestation singleton sheep fetuses (35).

In summary, we have shown that periconceptional undernutrition results in different effects on fetal and placental growth in late gestation, depending on whether it occurs before, after, or both before and after conception. These effects do not appear to be mediated via the fetal glucoseinsulin axis or the maternal or fetal HPAA in late gestation. Maternal undernutrition at different times around conception can have important effects on fetal growth trajectory that are not reflected in size in late gestation. Furthermore, maternal nutritional status in the preconception period alone alters fetal growth responses to stressors in late gestation, suggesting that nutrition in both periods is important for determining fetal growth trajectories, and that the effects are neither due solely to limitation of substrates for tissue accretion, nor to excess fetal glucocorticoid exposure at the time of undernutrition.

Acknowledgments. We thank the Liggins Institute's Fetal and Neonatal Physiology Group for invaluable contributions, and José Derraik for assistance with this manuscript.

\section{REFERENCES}

1. Godfrey K, Robinson S, Barker DJ, Osmond C, Cox V 1996 Maternal nutrition in early and late pregnancy in relation to placental and fetal growth. BMJ 312:410-414

2. Rao S, Yajnik CS, Kanade A, Fall CH, Margetts BM, Jackson AA, Shier R, Joshi S, Rege S, Lubree H, Desai B 2001 Intake of micronutrient-rich foods in rural Indian mothers is associated with the size of their babies at birth: Pune Maternal Nutrition Study. J Nutr 131:1217-1224

3. Roseboom T, de Rooij S, Painter R 2006 The Dutch famine and its long-term consequences for adult health. Early Hum Dev 82:485-491

4. Langley-Evans SC 2001 Fetal programming of cardiovascular function through exposure to maternal undernutrition. Proc Nutr Soc 60:505-513

5. Kwong WY, Wild AE, Roberts P, Willis AC, Fleming TP 2000 Maternal undernutrition during the preimplantation period of rat development causes blastocyst abnormalities and programming of postnatal hypertension. Development 127:41954202

6. Oliver MH, Hawkins P, Harding JE 2005 Periconceptional undernutrition alters growth trajectory, endocrine and metabolic responses to fasting in late gestation fetal sheep. Pediatr Res 57:591-598

7. Harding JE 1997 Periconceptual nutrition determines the fetal growth response to acute maternal undernutrition in fetal sheep of late gestation. Prenat Neonatal Med 2:310-319

8. Oliver MH, Hawkins P, Breier BH, Van Zijl PL, Sargison SA, Harding JE 2001 Maternal undernutrition during the periconceptual period increases plasma taurine levels and insulin response to glucose but not arginine in the late gestational fetal sheep. Endocrinology 142:4576-4579

9. Bloomfield FH, Oliver MH, Hawkins P, Holloway AC, Campbell M, Gluckman PD Harding JE, Challis JR 2004 Periconceptional undernutrition in sheep accelerates maturation of the fetal hypothalamic-pituitary-adrenal axis in late gestation. Endocrinology 145:4278-4285

10. Todd SE, Oliver MH, Jaquiery AL, Bloomfield FH, Harding JE 2009 Periconceptional undernutrition of ewes impairs glucose tolerance in their adult offspring. Pediatr Res 65:409-413

11. MacLaughlin SM, Walker SK, Roberts CT, Kleemann DO, McMillen IC 2005 Periconceptional nutrition and the relationship between maternal body weight changes in the periconceptional period and feto-placental growth in the sheep. J Physiol 565:111-124

12. MacLaughlin SM, Walker SK, Kleemann DO, Sibbons JP, Tosh DN, Gentili S, Coulter CL, McMillen IC 2007 Impact of periconceptional undernutrition on adrenal growth and adrenal insulin-like growth factor and steroidogenic enzyme expression in the sheep fetus during early pregnancy. Endocrinology 148:1911-1920

13. Gardner DS, Van Bon BW, Dandrea J, Goddard PJ, May SF, Wilson V, Stephenson $\mathrm{T}$, Symonds ME 2006 Effect of periconceptional undernutrition and gender on hypothalamic-pituitary-adrenal axis function in young adult sheep. J Endocrinol 190:203-212

14. Gardner DS, Pearce S, Dandrea J, Walker R, Ramsay MM, Stephenson T, Symonds ME 2004 Peri-implantation undernutrition programs blunted angiotensin II evoked baroreflex responses in young adult sheep. Hypertension 43:1290-1296

15. Harding JE 1997 Prior growth rate determines the fetal growth response to acute maternal undernutrition in fetal sheep of late gestation. Prenat Neonatal Med 2:300-309

16. Vatnick I, Schoknecht PA, Darrigrand R, Bell AW 1991 Growth and metabolism of the placenta after unilateral fetectomy in twin pregnant ewes. J Dev Physiol 15:351-356

17. Oliver MH, Harding JE, Breier BH, Evans PC, Gluckman PD 1993 Glucose but not a mixed amino acid infusion regulates plasma insulin-like growth factor-I concentrations in fetal sheep. Pediatr Res 34:62-65

18. Vickers MH, Casey PJ, Champion ZJ, Gravance CG 1999 IGF-I treatment increases motility and improves morphology of immature spermatozoa in the GH-deficient dwarf (dw/dw) rat. Growth Horm IGF Res 9:236-240

19. Rumball CW, Oliver MH, Thorstensen EB, Jaquiery AL, Husted SM, Harding JE, Bloomfield FH 2008 Effects of twinning and periconceptional undernutrition on late-gestation hypothalamic-pituitary-adrenal axis function in ovine pregnancy. Endocrinology 149:1163-1172

20. Rumball CW, Harding JE, Oliver MH, Bloomfield FH 2008 Effects of twin pregnancy and periconceptional undernutrition on maternal metabolism, fetal growth and glucose-insulin axis function in ovine pregnancy. J Physiol 586:1399-1411

21. Jaquiery AL, Oliver MH, Bloomfield FH, Connor KL, Challis JR, Harding JE 2006 Fetal exposure to excess glucocorticoid is unlikely to explain the effects of periconceptional undernutrition in sheep. J Physiol 572:109-118

22. Ronnenberg AG, Wang X, Xing H, Chen C, Chen D, Guang W, Guang A, Wang L, Ryan L, Xu X 2003 Low preconception body mass index is associated with birth outcome in a prospective cohort of Chinese women. J Nutr 133:3449-3455 
23. Abrams B, Selvin S 1995 Maternal weight gain pattern and birth weight. Obstet Gynecol 86:163-169

24. Duvekot JJ, Cheriex EC, Pieters FA, Menheere PP, Schouten HJ, Peeters LL 1995 Maternal volume homeostasis in early pregnancy in relation to fetal growth restriction. Obstet Gynecol 85:361-367

25. Nylund L, Lunell NO, Lewander R, Sarby B 1983 Uteroplacental blood flow index in intrauterine growth retardation of fetal or maternal origin. Br J Obstet Gynaecol 90:16-20

26. Redmer DA, Wallace JM, Reynolds LP 2004 Effect of nutrient intake during pregnancy on fetal and placental growth and vascular development. Domest Anim Endocrinol 27:199-217

27. Jaquiery AL, Oliver MH, Rumball C, Bloomfield FH, Harding JE 2009 Undernutrition before mating in ewes impairs the development of insulin resistance during pregnancy. Obstet Gynecol 114(4), in press

28. Rumball CW, Bloomfield FH, Harding JE 2008 Cardiovascular adaptations to pregnancy in sheep and effects of periconceptional undernutrition. Placenta 29:89-94

29. Lesage J, Bondeau B, Grino M, Breant B, Dupouy JP 2001 Maternal undernutrition during late gestation induces fetal overexposure to glucocorticoids and intrauterine growth retardation, and disturbs the hypothalamo-pituitary-adrenal axis in the newborn rat. Endocrinology 142:1692-1702
30. McMullen S, Osgerby JC, Thurston LM, Gadd TS, Wood PJ, Wathes DC, Michael AE 2004 Alterations in placental $11 \beta$-hydroxysteroid dehydrogenase $(11 \beta \mathrm{HSD})$ activities and fetal cortisol:cortisone ratios induced by nutritional restriction prior to conception and at defined stages of gestation in ewes. Reproduction 127:717-725

31. Connor KL, Challis JR, van Zijl PL, Rumball CW, Alix S, Jaquiery AL, Oliver MH, Harding JE, Bloomfield FH. Do alterations in placental $11 \beta$-hydroxysteroid dehydrogenase (11 $\beta$ HSD) activities explain differences in fetal hypothalamic pituitary adrenal (HPA) function following periconceptional undernutrition or twin conception in sheep? Reprod Sci, in press

32. Faichney GJ, White GA 1987 Effects of maternal nutritional status on fetal and placental growth and on fetal urea synthesis in sheep. Aust J Biol Sci 40:365-377

33. Bloomfield FH, Oliver MH, Hawkins P, Campbell M, Phillips DJ, Gluckman PD, Challis JR, Harding JE 2003 A periconceptional nutritional origin for noninfectious preterm birth. Science 300:606

34. Schieve LA, Cogswell ME, Scanlon KS 1999 Maternal weight gain and preterm delivery: differential effects by body mass index. Epidemiology 10:141-147

35. Edwards LJ, McMillen IC 2002 Impact of maternal undernutrition during the periconceptional period, fetal number, and fetal sex on the development of the hypothalamo-pituitary adrenal axis in sheep during late gestation. Biol Reprod 66:1562-1569 